\title{
ON A CLASS OF RIEMANN SURFACES ${ }^{1}$
}

\author{
R. D. M. ACCOLA
}

1. Introduction. In $1952 \mathrm{~L}$. Sario [1] introduced a class of Riemann surfaces, $O_{K D}$, which falls strictly between $O_{H D}$ and $O_{A D}$. The purpose of this paper is to investigate a class, $O_{H Q}$, which lies strictly between $O_{K D}$ and $O_{A D}$. A close examination of Sario's modular criterion for an $A D$-removable boundary $[2$, p. 285] shows this criterion to be sufficient for membership in $O_{H Q}$. It also turns out that $O_{H Q}$ is preserved under quasi-conformal mappings, while it is known that $O_{A D}$ is not. Since satisfying Sario's modular criterion is also a quasi-conformal invariant, the class $O_{H Q}$ seems more appropriate as a class determined by Sario's criterion.

This paper discusses these questions about $O_{H Q}$ and shows how surfaces of this class arise in other connections. Also Sario's criterion is used in a more restricted situation to produce surfaces where the generalized bilinear relation holds.

2. Criteria for membership in $O_{H Q}$. Let $\Gamma(W)$ stand for the Hilbert space of square integrable differentials on a Riemann surface $W .^{2}$ Let $\Gamma_{h}$ stand for the harmonic differentials in $\Gamma$, let $\Gamma_{h e}$ be the exact differentials in $\Gamma_{h}$, and let $\Gamma_{h o}=\Gamma_{h} \cap \Gamma_{h e}^{* \perp}$. Let $\Gamma_{h s e}$ be the differentials in $\Gamma_{h}$ which have vanishing periods over dividing cycles and let $\Gamma_{h m}$ $=\Gamma_{h} \cap \Gamma_{h s e}^{* \perp}$. If the genus of $W$ is finite then $\Gamma_{h m}=\Gamma_{h e} \cap \Gamma_{h o}$. In general, however, $\Gamma_{h m}$ is a proper subset of $\Gamma_{h e} \cap \Gamma_{h o}$ as the example below shows. A surface, $W$, is in Sario's class $O_{K D}$ if and only if $\Gamma_{h e}(W)$ $=\Gamma_{h m}(W)$. A close examination of Sario's modular criterion, which will be given below, shows that it insures that $\Gamma_{h e} \perp \Gamma_{h e}^{*}$; that is, $\Gamma_{h \theta} \subset \Gamma_{h o}$. We define $O_{H Q}$ to be the class of surfaces, $W$, for which $\Gamma_{h e}(W) \subset \Gamma_{h o}(W)$. If we define $\Gamma_{h q}=\Gamma_{h} \cap\left(\Gamma_{h o} \cap \Gamma_{h e}\right)^{* \perp}$ then $W \in O_{H Q}$ if and only if $\Gamma_{h e} \cap \Gamma_{h q}^{*}=0$. It follows easily that

$$
O_{K D} \subset O_{H Q} \subset O_{A D} \text {. }
$$

We remark, without proof, that if $\Gamma_{h e}(W)$ is finite dimensional then $W \in O_{H Q}$.

Presented to the Society, July 14, 1961; received by the editors October 1, 1962 and, in revised form, March 1, 1963.

1 This work was sponsored by the Office of Naval Research, Contract number Nonr 562 (31).

2 For a complete treatment of square integrable differentials on open surfaces the reader is referred to Ahlfors and Sario [1, Chapter V]. It will be assumed that the reader is familiar with this treatment. 
If $\omega$ is a closed square integrable differential then $\omega=\sigma+\tau$, where $\sigma$ is harmonic and $\tau$ can be approximated in the Dirichlet norm by exact differentials with compact support. ${ }^{3}$ If $W \in O_{H Q}$ and $\omega$ is exact then $\sigma$ is of class $\Gamma_{h o}$ and thus can be $D$-approximated by closed differentials with compact support. ${ }^{4}$ It follows that another criterion for $W$ to be of class $O_{H Q}$ is that every exact square integrable differential can be $D$-approximated by closed (but not necessarily exact) differentials which have compact support. ${ }^{5}$ By known facts concerning quasi-conformal mappings it follows that $O_{H Q}$ is preserved under quasi-conformal mappings.

Following the reasoning of Royden $[1$, p. 64$]$ it follows that $O_{H Q}$ is a proper subset of $O_{A D}$ since the latter class is not preserved under quasi-conformal mappings.

3. Sario's modular criterion. Let $\bar{\Omega}$ be a finite Riemann surface; that is, $\bar{\Omega}$ is a compact bordered Riemann surface whose interior is $\Omega$ and whose boundary we will denote by $\partial \Omega$. Let $\bar{G}$ be another finite surface included in $\Omega$. Then $\bar{\Omega}-G$ is a union of finite surfaces. Denote $\Omega-\bar{G}$ by $R$ and let $R=\bigcup_{i=1}^{n} R_{i}$, where each $R_{i}$ is a connected surface. Let $\partial R_{i}=\alpha_{i} \cup \beta_{i}$, where $\alpha_{i}=\partial R_{i} \cap \partial G$ and $\beta_{i}=\partial R_{i} \cap \partial \Omega$. Let $u_{i}$ be harmonic and bounded in $\bar{R}_{i}$ such that $u_{i}=0$ on $\alpha_{i}$ and $u_{i}=1$ on $\beta_{i}$. Let $\mu_{i}$ be the square of the Dirichlet norm of $d u_{i}$ and let $\mu_{\Omega, G}$ be the maximum of the $\mu_{i}$. By $\mu_{\Omega}$ we will denote the minimum of the $\mu_{\Omega, G}$ as $\bar{G}$ ranges over all admitted finite subsurfaces of $\Omega$ which have the same genus as $\Omega$.

The following lemma is very similar to Lemma 1, p. 153 of Accola [1].

LEMma. Suppose $v$ and $w$ are real harmonic functions on $\bar{\Omega}$. Let $\bar{G}$ be a finite subsurface as described above. Then there exists a surface $\Omega^{\prime}$ such that $\bar{G} \subset \Omega^{\prime} \subset \Omega$ and ${ }^{6}$

$$
\left|\left(d v, d w^{*}\right)_{\Omega^{\prime}}\right| \leqq \mu_{\Omega, G}\|d v\|_{R}\|d w\|_{R}
$$

Proof. Continue the notation already introduced in this section. Define $u$ to equal $u_{i}$ in $\bar{R}_{i}$ and to be zero in $\bar{G}$. Let $\Omega_{t}=\{p \in \bar{\Omega} \mid u(p)<t\}$ for $0 \leqq t \leqq 1$. Let $S_{t i}=\bar{R}_{i} \cap \partial \Omega_{t}$. If $\sigma=a d x+b d y$ let $\rho(\sigma)|d z|$ be the linear density $\sqrt{ }\left(|a|^{2}+|b|^{2}\right)|d z| .^{7}$ Then $\left(d v, d w^{*}\right)_{\Omega_{t}}=\int_{u=t} v d w$

${ }^{3}$ Ahlfors and Sario [1, p. 282]. We will call approximation in the Dirichlet norm, $D$-approximation.

${ }^{4}$ Ahlfors and Sario [1, p. 293].

5 If every $d u \in \Gamma$ is $D$-approximable by exact differentials with compact support then $W \in O_{K D}$. See Royden [1].

- Subscripts on inner products denote the region over which the integral is to be computed.

${ }^{7}$ Ahlfors and Sario [1, p. 220]. 
$=\sum_{i} \int_{S_{t i}} v d w$. By several applications of the Schwarz inequality we have

$$
\begin{aligned}
\left|\int_{S_{t i}} v d w\right| \leqq & \int_{S_{t i}}|d v| \int_{S_{t i}}|d w| \leqq \int_{S_{t i}} \rho(d v) d u_{i}^{*} \int_{S_{t i}} \rho(d w) d u_{i}^{*} \\
\leqq & \sqrt{ }\left(\int_{S_{t i}} \rho^{2}(d v) d u_{i}^{*} \int_{S_{t i}} d u_{i}^{*}\right) \\
& \cdot \sqrt{ }\left(\int_{S_{t i}} \rho^{2}(d w) d u_{i}^{*} \int_{S_{t i}} d u_{i}^{*}\right) .
\end{aligned}
$$

Since $\int_{S_{t i}} d u_{i}^{*}=\left\|d u_{i}\right\|^{2}$ we have upon summing over $i$

$$
\begin{aligned}
\left|\left(d v, d w^{*}\right)_{\Omega_{t}}\right| & \leqq \mu_{\Omega, G} \sum_{i} \sqrt{ }\left(\int_{S_{t i}} \rho^{2}(d v) d u_{i}^{*} \int_{S_{t i}} \rho^{2}(d w) d u_{i}^{*}\right) \\
& \leqq \mu_{\Omega, G} \sqrt{ }\left(\int_{\partial \Omega_{t}} \rho^{2}(d v) d u^{*} \int_{\partial \Omega_{t}} \rho^{2}(d w) d u^{*}\right)
\end{aligned}
$$

Now integrating between $t=0$ and $t=1$ we have

$$
\begin{aligned}
\min _{0 \leqq t \leqq 1} \mid & \left(d v, d w^{*}\right)_{\Omega_{t}} \mid \\
& \leqq \int_{0}^{1}\left|\left(d v, d w^{*}\right)_{\Omega_{t}}\right| d t \\
& \leqq \mu_{\Omega, G} \sqrt{ }\left(\int_{0}^{1} d t \int_{\partial \Omega_{t}} \rho^{2}(d v) d u^{*}\right) \sqrt{ }\left(\int_{0}^{1} d t \int_{\partial \Omega_{t}} \rho^{2}(d w) d u^{*}\right) \\
& =\mu_{\Omega, G}\|d v\|_{R}\|d w\|_{R} .
\end{aligned}
$$

Note in particular that if $d w=d v^{*}$ we have

$$
\|d v\|_{G}^{2} \leqq \mu_{\Omega, G}\|d v\|_{R}^{2}
$$

Thus if $\mu_{\Omega, G}$ is small and $v$ is the real part of an analytic function then the norm of $d v$ is mostly concentrated in $R$.

TheOREM (SARIO's modular CRITERION). Suppose $W$ admits two exhaustions $\left\{\Omega_{n}\right\}$ and $\left\{G_{n}\right\}$ such that $\bar{G}_{n} \subset \Omega_{n}$ and the sets $\left\{R_{n}\right\}$, $R_{n}=\Omega_{n}-\bar{G}_{n}$, are mutually disjoint. Let $\mu_{n}=\mu_{\Omega_{n} G_{n}}$. If $\sum_{n=1}^{\infty} \mu_{n}^{-1}=\infty$ then $W \in O_{H Q}$.

Proof. By the lemma we need only show that for some subsequence

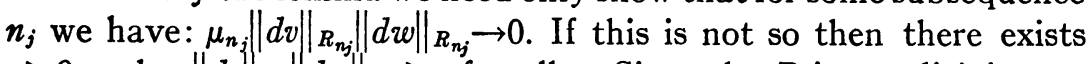
$\epsilon>0$ and $\mu_{n}\|d v\|_{R_{n}}\|d w\|_{R_{n}} \geqq \epsilon$ for all $n$. Since the $R_{n}$ 's are disjoint we reach the contradiction 


$$
\|d v\|\|d w\| \geqq \sum_{n}\|d v\|_{R_{n}}\|d w\|_{R_{n}} \geqq \sum_{n} \epsilon \mu_{n}^{-1}
$$

To show that $O_{K D}$ is a proper subset of $O_{H Q}$ we exhibit a surface where $\Gamma_{h e} \neq 0, \Gamma_{h m}=0$ and the surface satisfies Sario's modular criterion.

Let $W^{\prime}$ be the closed rectangle $\{0 \leqq x \leqq 1,-1 \leqq y \leqq 1\}$ in the $z$ plane minus slits $a_{n j}$ and $b_{n j}, n=2,3, \cdots, j=1,2, \cdots, 2^{n}-1$. The slits with second index $j$ lie on the line $x=j 2^{-n}$. For all $n, a_{n 1}=b_{n 1}$ and $|y| \leqq 1-2^{-n}$. For $a_{n j}, j=2,3, \cdots, 2^{n}-1$, we require $1-3 \cdot 2^{-n-1}$ $\leqq y \leqq 1-2^{-n}$. $b_{n j}$ is the reflection of $a_{n j}$ in the real axis. Let $W$ be $W^{\prime}$ doubled across all slits and the line $x=1$. The function $y$ on $W^{\prime}$ can be extended to $W$ to be symmetric since $d y^{*}=0$ along all the vertical slits. Thus $\Gamma_{h e}(W) \neq 0$. Since the complement of any compact set in $W$ is connected, $\Gamma_{h m}(W)=0$. Let $\Omega_{n}^{\prime}=W^{\prime} \cap\left\{2^{-n} \leqq x \leqq 1 ;|y|<1-2^{-n}\right\}$, and let $G_{n}^{\prime}=W^{\prime} \cap\left\{2^{-n} \leqq x \leqq 1 ;|y|<1-3 \cdot 2^{-n-1}\right\}$. Let $\Omega_{n}$ and $G_{n}$ be the doubles of $\Omega_{n}^{\prime}$ and $G_{n}^{\prime}$, respectively. Then $R_{n}=\Omega_{n}-\bar{G}_{n}$ is the union of $2\left(2^{n}-1\right)$ annuli each of modulus $1 / 4$. Thus Sario's criterion is satisfied for this surface.

It would be interesting to know if there are any other nonconstant Dirichlet bounded harmonic functions on this surface which are essentially different from $y$.

4. Doubling special kinds of bordered surfaces. Let $S O_{H B}$ (respectively, $S O_{H D}$ ) be the class of bordered surfaces for which the maximum principle holds with respect to the border for bounded (respectively, Dirichlet bounded) harmonic functions. It is known that $S O_{H B} \subset S O_{H D}$. If $\bar{W} \in S O_{H D}$ and $\hat{W}$ is the double of $\bar{W}$ then any $d u \in \Gamma_{h e}(\hat{W})$ must be symmetric with respect to the reflection of $\hat{W}$ that leaves $\partial W$ fixed. ${ }^{8}$ For if $d u$ were anti-symmetric then $u$ would be constant on $\partial W$ and so $d u$ would be zero. Since $d u^{*}$ is anti-symmetric it follows that $\Gamma_{h e}(\hat{W}) \perp \Gamma_{h e}(\hat{W})^{*}$ and therefore $W \in O_{H Q}$. A similar type of argument for bounded harmonic functions on the double, $\hat{W}$, of a surface of class $S O_{H B}$ shows that $\hat{W} \in O_{A B}$. Thus if $\bar{W} \in S O_{H B}$, then $\hat{W} \in O_{A B} \cap O_{H Q}$.

5. Remarks concerning the generalized bilinear relation. In Accola $[1$, p. 153$]$ a constant, also denoted $\mu_{\Omega}$, was introduced. If the constant as defined there is replaced by that defined at the beginning of $\$ 3$ of this paper then the proof of Lemma 1, p. 153 of the cited paper goes through without change. Thus if a surface $W$ admits a regular exhaustion $\left\{\Omega_{n}\right\}$ such that $\mu_{\Omega_{n}}$, new definition, is a bounded

\footnotetext{
${ }^{8}$ Ahlfors and Sario [1, p. 290].
} 
sequence, then the generalized bilinear relation holds with respect to the appropriate canonical homology basis. With this criterion it is easy to give examples of surfaces, $W$, for which the generalized bilinear relation holds and $W$ cannot be embedded in a parabolic surface. The surfaces will, however, be of class $O_{K D}$.

\section{REFERENCES}

R. Accola

1. The bilinear relation on open Reimann surfaces, Trans. Amer. Math. Soc. 96 (1960), 143-161.

L. AHLFORS AND L. SARIo

1. Riemann surfaces, Princeton Univ. Press, Princeton, N. J., 1960.

H. ROYDEN

1. On a class of null-bounded Riemann surfaces, Comment Math. Helv. 34 (1960), 52-66.

L. SARIO

1. An extremal method on arbitrary Riemann surfaces, Trans. Amer. Math. Soc. 73 (1952), 459-470.

2. Modular criteria on Riemann surfaces, Duke Math. J. 20 (1953), 279-286.

BROWN UNIVERSITY 